4

20

\title{
Effects of curcumin intake and aerobic exercise training on arterial compliance in
} postmenopausal women

\author{
Nobuhiko Akazawa ${ }^{\mathrm{a}}$, Youngju Choi ${ }^{\mathrm{b}}$, Asako Miyaki ${ }^{\mathrm{a}}$, Yoko Tanabe ${ }^{\mathrm{a}}$, Jun Sugawara ${ }^{\mathrm{c}}$, \\ Ryuichi Ajisaka $^{\mathrm{b}}$, Seiji Maeda ${ }^{\mathrm{b}}$
}

${ }^{\mathrm{a}}$ Graduate School of Comprehensive Human Science and ${ }^{\mathrm{b}}$ Faculty of Health and Sport Science, University of Tsukuba, 1-1-1 Tennodai, Tsukuba, Ibaraki 305-8577, Japan

${ }^{\mathrm{c}}$ Human Technology Research Institute, National Institute of Advanced Industrial Science and Technology (AIST), 1-1-1 Higashi, Tsukuba, Ibaraki 305-8566, Japan

\section{Corresponding author:}

Seiji Maeda, Ph.D.

Faculty of Health and Sport Sciences, University of Tsukuba

Tsukuba, Ibaraki 305-8577, Japan

Tel: +81 29-853-2683, Fax: +81 29-853-2986

E-mail:maeda@taiiku.tsukuba.ac.jp

1 Running title: Curcumin, exercise, and arterial compliance 
1 Abstract

2

3 Background: Reduction in arterial compliance with aging increases the risk of 4 cardiovascular disease. Lifestyle modification, particularly aerobic exercise and dietary 5 modification, has a favorable effect on vascular aging. Curcumin, a major component of 6 turmeric, is an anti-inflammatory agent. Therefore, it is plausible to hypothesize that 7 curcumin improves arterial compliance. We investigated the effects of curcumin ingestion 8 alone and in combination with aerobic exercise training on arterial compliance in 9 postmenopausal women.

10 Methods: A total of 51 postmenopausal women were assigned to 4 groups: placebo, curcumin, exercise and placebo $(\mathrm{Ex}+$ placebo $)$, and exercise and curcumin $(\mathrm{Ex}+$ curcumin $)$.

12 Curcumin or placebo was ingested orally for 8 weeks. The exercise groups underwent 13 moderate aerobic exercise training for 8 weeks.

14 Results: Carotid arterial compliance increased significantly in the curcumin, Ex + placebo, and Ex + curcumin groups, whereas no such changes were observed in the placebo control group. The magnitude of increases in carotid arterial compliance was the greatest in the Ex + curcumin group.

Conclusion: We concluded that curcumin ingestion improves carotid arterial compliance and that the combination of curcumin and aerobic exercise training was more efficacious in increasing central arterial compliance than either of these treatments alone in postmenopausal women.

Keywords: arterial stiffness, lifestyle modification, physical activity, turmeric 


\section{Introduction}

The large elastic arteries, such as the common carotid artery and the aorta, have the ability to buffer and cushion oscillation in blood pressure and blood flow. ${ }^{1}$ This compliant function of central arteries decreases with advancing age. ${ }^{2-3}$ Reduction in central arterial elasticity is an independent risk factor for cardiovascular disease. ${ }^{4}$ In addition, although arterial compliance in premenopausal women is greater than in age-matched men, this difference is lost in postmenopausal years, ${ }^{5-6}$ which suggests that postmenopausal women are at a higher risk of cardiovascular disease. ${ }^{7-8}$ We have previously demonstrated that regular aerobic exercise is clinically efficacious in preventing and treating decreased arterial compliance. ${ }^{9-10}$ Thus, it is therefore preferable to treat or prevent a decrease in arterial compliance without pharmacological therapies, e.g., lifestyle modification including exercise and/or diet.

Recent studies suggest that impairment of the arterial elastic properties is associated with chronic inflammatory disorders. ${ }^{11-12}$ Vlachopoulos et al. ${ }^{13}$ reported that acute systemic inflammation leads to deterioration of the central artery. Therefore, it is plausible that central arterial compliance improves with the aid of an anti-inflammatory agent. Curcumin is a polyphenolic molecule extracted from turmeric. ${ }^{14}$ Curcumin regulates various biochemical and molecular pathway by modulating several molecular targets, including transcription factors, cytolkines, enzymes, and gene regulating cell proliferation and apoptosis. ${ }^{15}$ As a results, curcumin has been shown to exert anti-inflammatory activity by binding to directly to pro-inflammatory molecules. ${ }^{15}$ Furthermore, it has been reported that curcumin may have protective effects against cardiovascular disease. ${ }^{16}$ However, the effect of curcumin ingestion on arterial compliance is unknown. Furthermore, although it is well known that aerobic exercise improves arterial compliance, ${ }^{3,9,10}$ the combined effect of exercise training and curcumin on arterial compliance has never been investigated. 
1 in combination with aerobic exercise training on central arterial compliance in

2 postmenopausal women. We hypothesized that curcumin ingestion increases arterial

3 compliance and the combination of curcumin and exercise training is more efficacious in 4 increasing arterial compliance than either treatment alone. To test these hypotheses, we used 5 a placebo-controlled study involving apparently healthy postmenopausal women.

6

\section{$7 \quad$ Methods}

8 Subjects.

9 A total of 51 healthy, sedentary postmenopausal women volunteered to participate. Subjects were assigned randomly to one of the following intervention groups: placebo group $(n=12)$, curcumin group $(n=12)$, exercise training with placebo group $(E x+$ placebo; $n=13)$, and exercise training with curcumin group $(\mathrm{Ex}+$ curcumin; $\mathrm{n}=14)$. Subjects were nonsmokers, nonobese, and free of cardiovascular disease as assessed by medical history. None of the subjects were taking cardiovascular-acting medications. All subjects gave their written informed consent to participate. All procedures were reviewed and approved by the ethical committee of the University of Tsukuba.

\section{Experimental design.}

All experiments proceeded in the morning after a 12-h overnight fast. Subjects abstained from alcohol and caffeine for at least $12 \mathrm{~h}$ and did not exercise for at least $24 \mathrm{~h}$ before beginning the experiment to avoid the potential acute effects of exercise. Measurements were taken in a quiet, temperature-controlled room $\left(24-26^{\circ} \mathrm{C}\right)$. After a resting period of at least 20 min, carotid arterial compliance, intima-media thickness (IMT), arterial blood pressure, and blood biochemistry were determined. After these measurements, peak oxygen consumption $\left(\mathrm{VO}_{2 \text { peak }}\right)$ was measured during incremental cycle ergometer exercise. These parameters were measured before and after each intervention.

\section{Curcumin ingestion.}


1 Subjects in the curcumin and Ex + curcumin groups ingested $150 \mathrm{mg}$ of curcumin

2 (Theracurumin, Theravalues Corporation, Tokyo) per day divided into 6 capsules. The

capsule with only starch (e.g., dextrin and maltose) was used as a placebo. Each subjects in the placebo and Ex + placebo groups ingested 6 placebo capsules. Curcumin or placebo was administered orally for 8 weeks. All subjects were instructed not to alter their dietary habits during the intervention period.

\section{Exercise intervention.}

Subjects in the Ex + placebo and Ex + curcumin groups underwent aerobic exercise training more than 3 days per week (2-3 supervised sessions and additional home-based training) for 8 weeks as previously described. ${ }^{10}$ Initially, subjects performed cycling and walking 30 $\mathrm{min} /$ day at a relatively low intensity $(60 \%$ of their individually determined maximal heart rate, which was evaluated by the maximal cycle exercise test). As their exercise tolerance improved, the intensity and time of aerobic exercise were increased to $40-60 \mathrm{~min} /$ day at an intensity of $70-75 \%$ of the maximal heart rate. Heart rate during exercise was evaluated by a digital pulse rate monitor (SM-66; Skynie, Tokyo, Japan). Subjects recorded their actual exercise and any additional physical activity on diary basis. Adherence to the exercise prescription was documented through the use of a uniaxial electrical accerometer (Lifecorder; KENZ, Nagoya, Japan) and physical activity logs as described previously. ${ }^{17}$ Subjects in the placebo and curcumin groups were instructed not to change their level of physical activity.

\section{Measurements}

Carotid Arterial Compliance. Carotid arterial compliance was determined using a combination of ultrasound imaging and simultaneous applanation tonometry of the common carotid artery. The common carotid artery was imaged B-mode using ultrasound (En Visor; Koinklijke Philips Electronics, Eindhoven, The Netherland) equipped with a high-resolution linear-array transducer $(7.5 \mathrm{~Hz})$. Diameters were measured from the intima of the far wall to the media-adventitia of the near wall. Pulsatile changes in the common carotid artery 
1 diameter were analyzed 1 to $2 \mathrm{~cm}$ proximal to the bifurcation. Carotid arterial pressure

2 waveforms were obtained with arterial applanation tonometry incorporating an array of 15 3 micropiezoresistive transducers (FormPWV/ABI; Colin Medical Technology, Komaki, 4 Japan), ${ }^{18}$ and were calibrated by equating the carotid mean arterial and diastolic blood 5 pressure to the brachial mean arterial and diastolic blood pressure. The arterial lumen 6 diameter at minimal diastolic relaxation and maximal systolic expansion of the vessel was 7 measured at 3 points per frame and then averaged. Each parameter was averaged over 10-15 8 continuous beats and statistically analyzed. Arterial compliance was obtained using the 9 following equation:

$$
[(\mathrm{D} 1-\mathrm{D} 0) / \mathrm{D} 0] /[2(\mathrm{P} 1-\mathrm{P} 0) \pi \mathrm{D} 0]
$$

where D1 and D0 are the maximal and minimum arterial diameters, and P1 and P0 are the highest and lowest blood pressures respectively. In addition to arterial compliance, the $\beta$-stiffness index was analyzed using the following equation:

$$
\ln (\mathrm{P} 1 / \mathrm{P} 0) /[(\mathrm{D} 1-\mathrm{D} 0) / \mathrm{D} 0]
$$

The $\beta$-stiffness index provided an index of arterial compliance adjusted for distending pressure. $^{19}$

Carotid Artery Intima-Media Thickness. Carotid artery IMT was measured from the images derived from the same ultrasound machine (En Visor; Koinklijke Philips Electronics, Eindhoven, The Netherland) as previously described. ${ }^{20}$ Carotid IMT was defined as distance from the leading edge of the lumen-intima interface. Lumen diameter was defined as the distance between the lumen and intima, and a near-wall boundary, corresponding to the interface of the adventitia and media. These measurements were made at end diastole. At least 10 measurements of IMT were taken at each segment, and the mean values were used for analysis.

Blood Chemistry. A blood sample was collected from the antecubital vein after overnight fasting. Serum concentrations of cholesterol and triglyceride were determined using standard 
1

2

3 Peak Oxygen Consumption. $\dot{\mathrm{VO}}_{2 \text { peak }}$ was measured during incremental cycle ergometer 4

enzymatic techniques.

exercise by using online computer-assisted circuit spirometry (AE280; Minato Medical Science, Osaka, Japan). All subjects underwent an incremental exercise test (after 2 min at 40 $\mathrm{W}$, with $20 \mathrm{~W}$ increases every $2 \mathrm{~min}$ ) until volitional exhaustion. $\mathrm{VO}_{2 \text { peak }}$ was defined at the highest value recorded during the test. Heart rate and rating of perceived exertion ${ }^{21}$ were measured throughout the exercise, and the total exercise duration required to reach exhaustion was recorded.

\section{Statistical analyses}

To determine the effect of each intervention on all outcome measures, repeated measures analysis of variance was used. When indicated by a significant main effect on intervention, specific mean comparisons were performed to identify significant within each intervention. In the case of a significant F-value, a post-hoc test (the Bonferroni test) was used to identify significant differences among mean values. All data are reported as means $\pm \mathrm{SE}$. Statistical significant was set a priori at $P<0.05$ for all comparisons.

\section{Results}

In the exercise groups $(\mathrm{Ex}+$ placebo and $\mathrm{Ex}+$ curcumin $)$, the average frequency and time of the exercise training were similar. The average frequency of the exercise training was $4.7 \pm 0.3$ days/week $(E x+$ placebo $)$ and $4.9 \pm 0.3$ days/week $(E x+$ curcumin $)(p=0.776)$, and the average time of the exercise training was $43.0 \pm 1.6 \mathrm{~min} /$ day $(\mathrm{Ex}+$ placebo $)$ and $49.5 \pm$ $3.8 \mathrm{~min} /$ day $(\mathrm{Ex}+$ curcumin $)(\mathrm{p}=0.136)$. There were no significant differences in compliance/adherence to the placebo and curcumin ingestion regimen between the 4 groups $(95 \% \pm 3 \%$ in placebo; $99 \% \pm 1 \%$ in curcumin; $98 \% \pm 1 \%$ in $\mathrm{Ex}+$ placebo; $98 \% \pm 1 \%$ in Ex + curcumin). 
1

Table 1 shows the baseline characteristics of the study participants. Before the intervention, there were no significant differences in any of the variables among groups. Body weight and body mass index decreased in the Ex + curcumin group $(\mathrm{P}<0.05)$. High-density lipoprotein cholesterol increased significantly after the intervention in the Ex + placebo group $(\mathrm{P}<0.05)$. There were no significant changes in low-density lipoprotein cholesterol and triglyceride level with any of the intervention. Absolute and relative $\dot{\mathrm{VO}}_{2 \text { peak }}$ in the exercise groups (both Ex + placebo and Ex + curcumin groups) increased significantly after the intervention $(\mathrm{P}<0.05)$.

As shown in Table 2, there were no statistically significant differences in the baseline hemodynamic parameters at rest among the groups before intervention. Heart rate did not change in any of the group. After 8 weeks of intervention, brachial and carotid systolic blood pressure decreased in the curcumin, Ex + placebo, and Ex + curcumin groups $(\mathrm{P}<$ 0.05). Brachial diastolic blood pressure significantly decreased in the Ex + curcumin group ( $P$ $<0.05)$. Brachial pulse pressure significantly decreased in the $\mathrm{Ex}+$ placebo group $(\mathrm{P}<0.05)$. IMT did not change before and after each intervention in all groups. Carotid pulse pressure and $B$-stiffness index significantly decreased in the Ex + placebo and Ex + curcumin groups $(\mathrm{P}<0.05)$.

There was no significant difference in the baseline carotid arterial compliance among the groups (Fig. 1). After 8 weeks of intervention, carotid arterial compliance increased significantly in the curcumin, Ex + placebo, and Ex + curcumin groups. There was no significant change in carotid arterial compliance in the placebo control group. The percent change in carotid arterial compliance was significantly greater in the Ex + curcumin group than in the placebo group (Fig. 2). On the other hand, the percent change in the curcumin group or the Ex + placebo group statistically did not differ compared with the placebo group (Fig. 2). 


\section{Discussion}

The main findings of the present investigation were as follows. A regular ingestion of curcumin significantly increased carotid arterial compliance in postmenopausal women. The magnitude of improvement by curcumin was similar to that of exercise training alone. Moreover, the combination of exercise training and curcumin ingestion led to a greater improvement in arterial compliance compared to that achived with either treatment alone. These results suggest that a combination of exercise and curcumin can have a strong positive effect on arterial compliance. as a spice to add flavor and yellow, coloring to food. Curcumin is known to anti-inflammatory effects in addition to acting as an anti-carcinogenic and neuroprotective agent. $^{22-24}$ To our knowledge, the effects of curcumin an arterial compliance have never been studied. In the present study, we revealed for the first time that central arterial compliance increased after 8 weeks of the curcumin ingestion in postmenopausal women. Furthermore, the magnitude of improvement achieved by curcumin treatment was comparable to that obtained with exercise treatment $(10.1 \% \pm 4.5 \%$ vs. $10.0 \% \pm 3.6 \%)$. This finding suggests that the favorable effect of curcumin can be a primary therapeutic approach for cardiovascular disease in postmenopausal women.

In our previous study, we investigated the effect of exercise training and curcumin ingestion on central arterial hemodynamics, i.e., wave reflection and central blood pressure. ${ }^{25}$ Wave reflection and central blood pressure were not improved by exercise training alone or curcumin ingestion alone. These parameters improved in the combined exercise training with curcumin ingestion. On the other hand, the present study investigated the effect of curcumin on arterial compliance. We demonstrated for the first time that curcumin ingestion alone increased arterial compliance. In addition, the magnitude of increase in carotid arterial compliance was the greatest in the Ex + curcumin group among 4 groups. Therefore, these 
results suggest that the combination of exercise and curcumin may more effectively increase arterial compliance than either treatment alone, although curcumin ingestion alone or exercise training alone increased arterial compliance.

The mechanism responsible for the curcumin ingestion induced improvement in arterial compliance is unclear in this study. Arterial elastic property is associated with inflammatory cytokines, such as tumor necrosis factor-alpha (TNF- $\alpha){ }^{12}$ Curcumin exerts anti-inflammatory effects by inhibiting the expression of cytokines including TNF- $\alpha .{ }^{26}$ Therefore, the effect of curcumin on arterial compliance may be mediated by suppression of inflammation via downregulating of TNF- $\alpha$. However, we did not measure any inflammatiory cytokines in this study. Further studies are warranted to clarify the mechanism underlying the effect of curcumin on arterial compliance.

Most measures of arterial compliance are somewhat dependent on arterial pressure. It is possible that the improvement in the arterial compliance by our treatment of curcumin ingestion and exercise training may be mediated by the corresponding changes in arterial blood pressure. To address this possibility, we calculated the $\beta$-stiffness index of arterial compliance adjusted for distending pressure. The results indicated that improvement in arterial compliance after the exercise intervention remained statistically significant even when the data were expressed as the $\beta$-stiffness index. However, the change in the $\beta$-stiffness index after curcumin intervention did not attain statistical significance. Thus, the improvement in arterial compliance with curcumin may be effected partly by the epiphenomenon of blood pressure change.

We observed that the greatest change in arterial compliance was achieved with a combination of curcumin ingestion and exercise training. This result may be attributable to physiological mechanism by which exercise training and curcumin increase arterial compliance. Aerobic exercise training results in a decrease in of endothelin-1 production ${ }^{27}$ 
1

and $\alpha$-adrenergic vasoconstrictor tone. ${ }^{28}$ To the best of our knowledge, there have been no reports that curcumin positively influence these mechanisms. Therefore, curcumin and exercise training are likely to have different physiological mechanisms to improve the elastic property of the large artery. This may be the reason why combining exercise training with curcumin ingestion appears more effective than a single intervention.

It is unclear that body weight and BMI decrease only in the Ex + curcumin group. Little is known to the effect of curcumin on body composition. An animal study suggests that curcumin increases fatty acid oxidation and reduces fatty acid esterification, resulting in catabolism in adipose tissue and body weight reduction in obese mice. ${ }^{29}$ However, our present study was not observed weight and BMI reduction by curcumin supplementation alone in postmenopausal women. Exercise training alone also did not decrease body weight and BMI. On the other hand, the combination of exercise training with curcumin ingestion decreases body weight and BMI. It is possible that fatty acid oxidation is promoted by the combination of curcumin ingestion and exercise training in postmenopausal women.

6

In conclusion, the present study demonstrated that curcumin ingestion alone increases arterial compliance in postmenopausal women and that combining curcumin ingestion with aerobic exercise training more effectively increases arterial compliance than curcumin ingestion or aerobic exercise training alone. Regular curcumin ingestion and aerobic exercise may be effective lifestyle modifications for minimizing and reversing the loss of carotid arterial compliance with advancing age in women.

\section{Acknowledgments}

This work was supported by Grants-in-Aid for Scientific Research 21300234 and 2160179 from Japan Society for the Promotion of Science. 


\section{Conflict of interest}

2

We have no financial, consultant, institutional and other relationships that might lead

3 to bias or a conflict of interest.

4 


\section{References}

1. Nichols WW, O'Rourke MF. Mc'Donald's Blood Flow in Arteries, Theoretical, Experimental, and Clinical Principles, 5th ed. London, Arnold, 2005

2. Lakatta EG. Cardiovascular aging in health. Clin Geriatr Med 2000; 16: 419-444.

3. Tanaka H, Dinenno FA, Monahan KD, Clevenger CM, DeSouza CA, Seals DR. Aging, habitual exercise, and dynamic arterial compliance. Circulation 2000; 102: 1270-1275.

4. Najjar SS, Scuteri A, Lakatta EG. Arterial aging: is it an immutable cardiovascular risk factor? Hypertension 2005; 46: 454-462.

5. Karpanou EA, Vyssoulis GP, Papakyriakou SA, Toutouza MG, Toutouzas PK. Effects of menopause on aortic root function in hypertensive women. J Am Coll Cardiol 1996; 28: $1562-1566$.

6. Staessen JA, van der Heijden-Spek JJ, Safar ME, Den Hond E, Gasowski J, Fagard RH, et al. Menopause and the characteristics of the large arteries in a population study. J Hum Hypertens 2001; 15: 511-518.

7. La Vecchia C. Sex hormones and cardiovascular risk. Hum Reprod 1992; 7: 162-167.

8. Zaydun G, Tomiyama H, Hashimoto H, Arai T, Koji Y, Yambe M, et al. Menopause is an independent factor augmenting the age-related increase in arterial stiffness in the early postmenopausal phase. Atherosclerosis 2006; 184: 137-142.

9. Maeda S, Sugawara J, Yoshizawa M, Otsuki T, Shimojo N, Jesmin S, et al. Involvement of endothelin-1 in habitual exercise-induced increase in arterial compliance. Acta Physiol 
1

2

3

4

5

6

7

10. Yoshizawa M, Maeda S, Miyaki A, Misono M, Choi Y, Shimojo N, et al. Additive beneficial effects of lactotripeptides and aerobic exercise on arterial compliance in postmenopausal women. Am J Physiol Heart Circ Physiol 2009; 297: H1899-1903.

11. Amar J, Ruidavets JB, Bal dit Sollier C, Bongard V, Boccalon H, Chamontin B, et al. CD14 C(-260)T gene polymorphism, circulating soluble CD14 levels and arteriosclerosis. J Hypertens 2004; 22: 1523-1528

12. Mahmud A, Feely J. Arterial stiffness is related to systemic inflammation in essential hypertension. Hypertension 2005; 46: 1118-1122.

13. Vlachopoulos C, Dima I, Aznaouridis K, Vasiliadou C, Ioakeimidis N, Aggeli C, et al. Acute systemic inflammation increases arterial stiffness and decreases wave reflections in healthy individuals. Circulation 2005; 112: 2193-2200.

14. Goel A, Kunnumakkara AB, Aggarwal BB. Curcumin as "Curecumin": from kitchen to clinic. Biochem Pharmacol 2008; 75: 787-809.

15. Gupta SC, Patchva S, Koh W, Aggarwal BB. Discovery of curcumin, a component of golden spice, and its miraculous biological activities. Clin Exp Pharmacol Physiol 2012; 39: 283-299.

16. Wongcharoen W, Phrommintikul A. The protective role of curcumin in cardiovascular diseases. Int J Cardiol 2009; 133: 145-151. 
1 17. Sugawara J, Otsuki T, Tanabe T, Hayashi K, Maeda S, Matsuda M. Physical activity duration, intensity, and arterial stiffening in postmenopausal women. Am J Hypertens 2006; 19: 1032-1036.

4

18. Cortez-Cooper MY, Supak JA, Tanaka H. A new device for automatic measurements of arterial stiffness and ankle-brachial index. Am J Cardiol 2003; 91: 1519-1522.

22. Sharma RA, Gescher AJ, Steward WP. Curcumin: the story so far. Eur J Cancer 2005; 41: 1955-1968.

23. Maheshwari RK, Singh AK, Gaddipati J, Srimal RC. Multiple biological activities of curcumin: a short review. Life Sci 2006; 78: 2081-2087.

24. Jiang J, Wang W, Sun YJ, Hu M, Li F, Zhu DY. Neuroprotective effect of curcumin on focal cerebral ischemic rats by preventing blood-brain barrier damage. Eur J Pharmacol 2007; 561: 54-62. 
25. Sugawara J, Akazawa N, Miyaki A, Choi Y, Tanabe Y, et al. Effect of endurance exercise training and curcumin intake on central arterial hemodynamics in postmenopausal women: pilot study. Am J Hypertens 2012; 25: 651-656.

26. Aggarwal S, Ichikawa H, Takada Y, Sandur SK, Shishodia S, Aggarwal BB. Curcumin (diferuloylmethane) down-regulates expression of cell proliferation and antiapoptotic and metastatic gene products through suppression of IkappaBalpha kinase and Akt activation. Mol Pharmacol 2006; 69: 195-206.

27. Maeda S, Tanabe T, Otsuki T, Sugawara J, Iemitsu M, Kuno S, et al. Aerobic exercise training reduce plasma endothelin-1 concentration in older women. J Appl Physiol 2003; 95: $336-341$

28. Sugawara J, Komine H, Hayashi K, Yoshizawa M, Otsuki T, Shimojo N, et al. Reduction in alpha-adrenergic receptor-mediated vascular tone contributes to improved arterial compliance with endurance training. Int J Cardiol 2009; 135: 346-352.

29. Ejaz A, Wu D, Kwan P, Meydani M. Curcumin inhibits adipogenesis in 3T3-L1 adipocytes and angiogenesis and obesity in C57/BL mice. J Nutr 2009; 139: 919-925. 


\section{$1 \quad$ Figure legends}

2 Figure.1 Carotid arterial compliance before and after intervention. Data are expressed 3 as mean $\pm \mathrm{SE}$. $* \mathrm{P}<0.05$ before vs. after intervention.

4

5 Figure.2 Percent changes in arterial compliance in response to intervention. Data are 6 expressed as mean $\pm \mathrm{SE} .{ }^{*} \mathrm{P}<0.05$ placebo vs. exercise + curcumin $(\mathrm{Ex}+$ curcumin $)$. 7 
Table 1. Selected subject characteristics

\begin{tabular}{|c|c|c|c|c|c|c|c|c|c|}
\hline \multirow[b]{2}{*}{ Age, years } & \multirow[b]{2}{*}{ Before } & \multicolumn{2}{|c|}{ Placebo } & \multicolumn{2}{|c|}{ Curcumin } & \multicolumn{2}{|c|}{ Ex + placebo } & \multicolumn{2}{|c|}{ Ex + Curcumin } \\
\hline & & 58 & \pm 1 & 60 & \pm 2 & 59 & \pm 2 & 60 & \pm 1 \\
\hline Height, $\mathrm{cm}$ & Before & 156 & \pm 2 & 155 & \pm 2 & 154 & \pm 1 & 157 & \pm 1 \\
\hline \multirow[t]{2}{*}{ Weight, $\mathrm{kg}$} & Before & 52.1 & \pm 1.9 & 52.6 & \pm 2.5 & 52.2 & \pm 1.5 & 56.6 & \pm 1.8 \\
\hline & After & 52.3 & \pm 1.8 & 52.7 & \pm 2.5 & 52.1 & \pm 1.5 & 55.6 & $\pm 1.5^{*}$ \\
\hline \multirow[t]{2}{*}{ Body mass index, $\mathrm{kg} / \mathrm{m}^{2}$} & Before & 21.5 & \pm 0.8 & 21.9 & \pm 0.7 & 22.1 & \pm 0.5 & 23.2 & \pm 1.8 \\
\hline & After & 21.6 & \pm 0.7 & 22.0 & \pm 0.7 & 22.1 & \pm 0.6 & 22.7 & $\pm 0.8^{*}$ \\
\hline \multirow[t]{2}{*}{ HDL cholesterol, mmol/L } & Before & 1.73 & \pm 0.11 & 1.58 & \pm 0.13 & 1.69 & \pm 0.07 & 1.78 & \pm 0.10 \\
\hline & After & 1.81 & \pm 0.12 & 1.61 & \pm 0.13 & 1.85 & $\pm 0.08^{*}$ & 1.89 & \pm 0.12 \\
\hline \multirow[t]{2}{*}{ LDL cholesterol, $\mathrm{mmol} / \mathrm{L}$} & Before & 3.45 & \pm 0.12 & 3.65 & \pm 0.21 & 3.56 & \pm 0.19 & 3.64 & \pm 0.15 \\
\hline & After & 3.42 & \pm 0.10 & 3.98 & \pm 0.20 & 3.57 & \pm 0.18 & 3.74 & \pm 0.19 \\
\hline \multirow[t]{2}{*}{ Triglyceride, $\mathrm{mmol} / \mathrm{L}$} & Before & 1.15 & \pm 0.15 & 1.66 & \pm 0.29 & 1.16 & \pm 0.11 & 1.15 & \pm 0.13 \\
\hline & After & 1.24 & \pm 0.15 & 1.56 & \pm 0.32 & 0.99 & \pm 0.12 & 1.16 & \pm 0.18 \\
\hline \multirow[t]{2}{*}{$\mathrm{VO}_{2}$ peak, $\mathrm{ml} / \mathrm{min}$} & Before & 1312 & \pm 78 & 1179 & \pm 58 & 1282 & \pm 66 & 1390 & \pm 79 \\
\hline & After & 1221 & \pm 56 & 1175 & \pm 62 & 1394 & $\pm 73^{*}$ & 1474 & $\pm 82 *$ \\
\hline \multirow[t]{2}{*}{$\dot{\mathrm{VO}}_{2}$ peak, $\mathrm{ml} / \mathrm{kg} / \mathrm{min}$} & Before & 25.6 & \pm 2.0 & 22.6 & \pm 0.9 & 24.7 & \pm 1.2 & 24.5 & \pm 1.2 \\
\hline & After & 23.5 & \pm 1.2 & 22.4 & \pm 0.8 & 26.9 & $\pm 1.4^{*}$ & 26.5 & $\pm 1.2^{*}$ \\
\hline
\end{tabular}

Values are means $\pm \mathrm{SE}$. HDL; high density lipoprotein, $\mathrm{LDL}$; low density lipoprotein, $\dot{\mathrm{VO}}_{2 \text { peak }}$; peak oxygen consumption. ${ }^{*} \mathrm{P}<0.05$ vs. before intervention. 
Table 2. Hemodynamic parameter before and after intervention

\begin{tabular}{|c|c|c|c|c|c|c|c|c|c|c|c|c|}
\hline \multirow[b]{2}{*}{ Heart rate, beats/min } & \multirow[b]{2}{*}{ Before } & \multicolumn{3}{|c|}{ Placebo } & \multicolumn{3}{|c|}{ Curcumin } & \multicolumn{2}{|c|}{ Ex + placebo } & \multicolumn{3}{|c|}{ Ex + Curcumin } \\
\hline & & 64 & \pm & 3 & 61 & \pm & 2 & 59 & \pm 1 & 60 & \pm & 2 \\
\hline & After & 62 & \pm & 2 & 59 & \pm & 2 & 57 & \pm 1 & 57 & \pm & 1 \\
\hline \multirow[t]{2}{*}{ Brachial SBP, mmHg } & Before & 114 & \pm & 4 & 123 & \pm & 5 & 112 & \pm 3 & 118 & \pm & 4 \\
\hline & After & 114 & \pm & 4 & 119 & \pm & $4^{*}$ & 108 & $\pm 3^{*}$ & 113 & \pm & $4^{*}$ \\
\hline \multirow[t]{2}{*}{ Brachial DBP, mmHg } & Before & 71 & \pm & 3 & 72 & \pm & 4 & 69 & \pm 2 & 71 & \pm & \\
\hline & After & 71 & \pm & 3 & 69 & \pm & 3 & 68 & \pm 2 & 67 & \pm & $3 *$ \\
\hline \multirow[t]{2}{*}{ Brachial PP, mmHg } & Before & 42 & \pm & 2 & 51 & \pm & 2 & 43 & \pm 3 & 47 & \pm & 2 \\
\hline & After & 43 & \pm & 2 & 50 & \pm & 2 & 40 & $\pm 2^{*}$ & 45 & \pm & 2 \\
\hline \multirow[t]{2}{*}{ Caroid SBP, mmHg } & Before & 103 & \pm & 3 & 112 & \pm & 5 & 103 & \pm 3 & 107 & \pm & 4 \\
\hline & After & 104 & \pm & 3 & 108 & \pm & $4^{*}$ & 99 & $\pm 3^{*}$ & 102 & \pm & $4^{*}$ \\
\hline \multirow[t]{2}{*}{ Carotid PP, mmHg } & Before & 32 & \pm & 2 & 40 & \pm & 2 & 34 & \pm 3 & 37 & \pm & 2 \\
\hline & After & 33 & \pm & 2 & 39 & \pm & 2 & 32 & $\pm 2^{*}$ & 35 & \pm & $2^{*}$ \\
\hline \multirow[t]{2}{*}{$\mathrm{IMT}, \mathrm{mm}$} & Before & 0.53 & \pm & 0.01 & 0.55 & \pm & 0.03 & 0.55 & \pm 0.03 & 0.52 & \pm & 0.02 \\
\hline & After & 0.53 & \pm & 0.01 & 0.55 & \pm & 0.03 & 0.54 & \pm 0.03 & 0.52 & \pm & 0.02 \\
\hline \multirow[t]{2}{*}{$\beta$-stiiffness, $U$} & Before & 7.6 & \pm & 0.4 & 7.8 & \pm & 0.6 & 8.3 & \pm 0.6 & 8.0 & \pm & 0.5 \\
\hline & After & 7.9 & \pm & 0.4 & 7.4 & \pm & 0.4 & 7.5 & $\pm 0.5^{*}$ & 7.2 & \pm & $0.4^{*}$ \\
\hline
\end{tabular}

Values are means \pm SE. SBP; systolic blood pressure, DBP; diastolic blood pressure, PP; pulse pressure, IMT; intima-media thickness. ${ }^{*} \mathrm{P}<0.05$ vs. before intervention. 
Figure 1

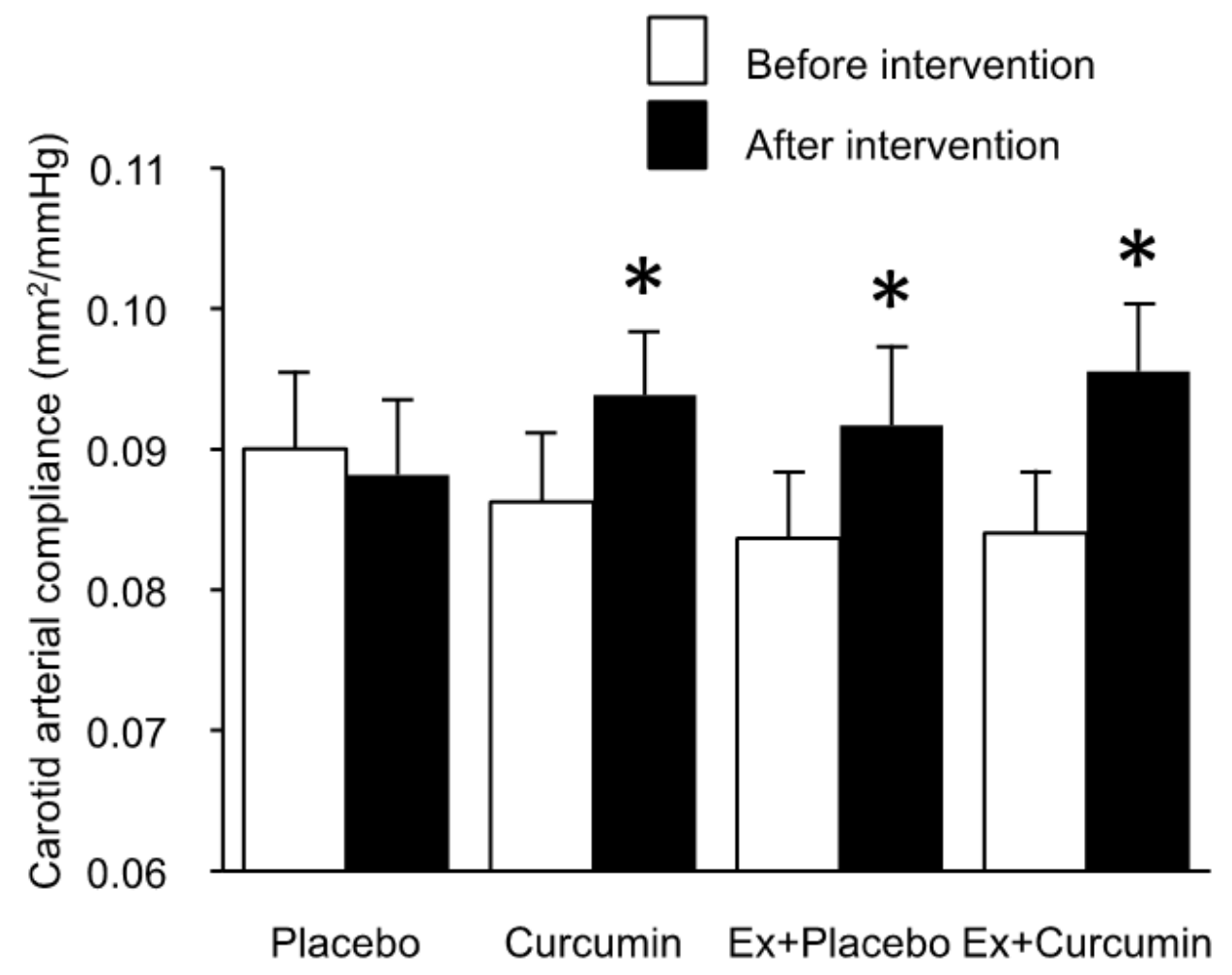


Figure 2

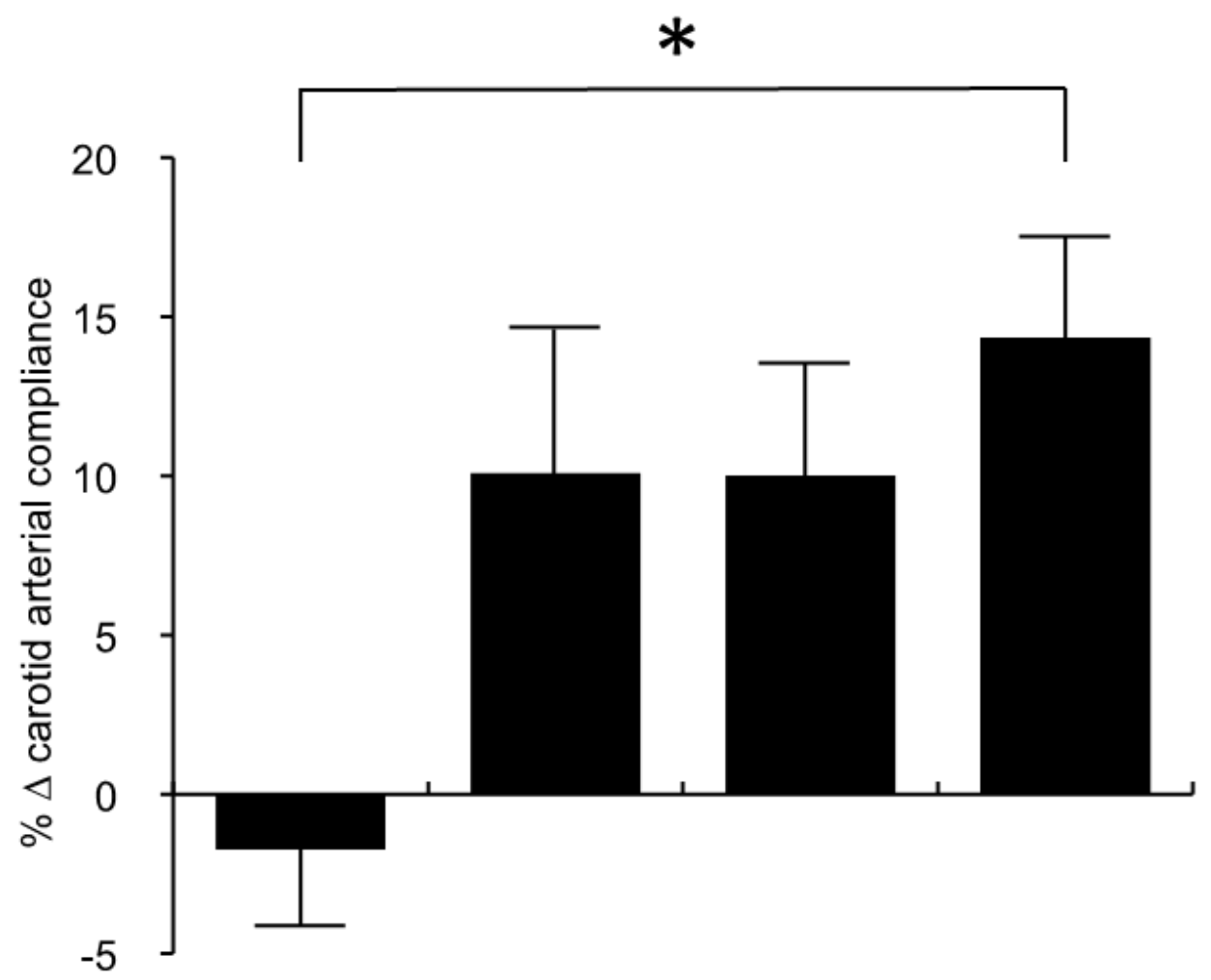

Placebo Curcumin Ex+Placebo Ex+Curcumin 